\title{
Identificando os desafios e as boas práticas para o transporte urbano de cargas, por meio de uma revisão bibliográfica sistemática
}

\author{
Cíntia Machado de Oliveira1 ${ }^{1}$ Márcio de Almeida D’Agosto², Ana Lucia Dorneles de Mello³, Fabio dos Santos \\ Gonçalves ${ }^{4}$, Daniel Neves Schmitz Gonçalves ${ }^{5}$ e Fabiana do Couto Assumpção ${ }^{6}$
}

\begin{abstract}
Resumo: Objetivou-se com este estudo, identificar, mediante uma revisão bibliográfica sistemática, os desafios enfrentados pelo transporte urbano de carga e as boas práticas que podem ser adotadas, visando os aspectos socioambientais. Identificaram-se também os agentes de implantação das boas práticas (poder público ou iniciativa privada). Como contribuição, apontam-se a elaboração de um procedimento de revisão bibliográfica sistemática, que foi estabelecido, exclusivamente, para este estudo e a identificação de 26 boas práticas capazes de apoiar a solução de 16 desafios do TUC. O poder público, a iniciativa privada e ambos os agentes podem solucionar 31\%, $44 \%$ e $25 \%$ dos desafios, respectivamente, atuando por meio de $27 \%$, $65 \%$ e $8 \%$ das práticas. Verificou-se que $81 \%$ das boas práticas podem contribuir positivamente, em relação aos aspectos econômicos. A pesquisa limitou-se a uma revisão bibliográfica. Recomenda-se que trabalhos futuros considerem uma pesquisa de campo para verificar se tais desafios e boas práticas identificados fazem parte da realidade das cidades.
\end{abstract}

Palavras-chave: revisão bibliográfica sistemática, transporte urbano de carga.

\begin{abstract}
This purpose of this paper is to identify, through a systematic literature review, the challenges faced in urban freight transportation and best practices that could be implemented in the field, considering social and environmental aspects. The research also identifies the actors (in public and private sectors) which could be responsible for implementing such practices. Moreover, the paper proposes a procedure for conducting systematic literature reviews, established exclusively for this study. The conclusions identify 26 best practices capable of supporting the solution of 16 challenges. Results indicate that $31 \%$ of these challenges could be solved by public actors, $44 \%$ by the private sector and $25 \%$ of them by both agents, working, respectively, in $27 \%, 65 \%$ and $8 \%$ of the practices. Besides, it was identified that $81 \%$ of the best practices can contribute positively, considering economic aspects. The research was limited to scientific papers. Therefore, it is recommended that future studies consider a field survey to confirm that the challenges and good practices identified are part of the reality of cities.
\end{abstract}

Keywords: systematic literature review, urban freight transport.

\section{INTRODUÇÃO}

No mundo, o transporte urbano de carga (TUC) tem importante papel no desenvolvimento das cidades e, em países em desenvolvimento, essa tendência é ainda mais evidente (Ibeas et al., 2012) pela concentração de grande massa populacional em áreas urbanas, o que promove uma demanda por transporte ainda maior nessas regiões (Allen et al., 2008).

Comparado ao transporte urbano de passageiro, o TUC tem um impacto significativo em relação aos aspectos sociais, ambientais e econômicos, sobretudo com relação aos congestionamentos, a poluição atmosférica, a emissão

\footnotetext{
1 Cíntia Machado de Oliveira1, Departamento de Engenharia de Transportes, UFRJ/COPPE. (cintia.machado.oliveira.1@gmail.com)

2 Márcio de Almeida D’Agosto, Departamento de Engenharia de

Transportes, UFRJ/COPPE. (dagosto@pet.coppe.ufrj.br)

${ }_{3}$ Ana Lucia Dorneles de Mello, Departamento de Engenharia de

Transportes, UFRJ/COPPE. (anadmello1@gmail.com)

${ }^{4}$ Fabio dos Santos Goncalves1, Departamento de Engenharia de

Transportes, UFRJ/COPPE. (fabio.goncalves@pet.coppe.ufr.br)

5 Daniel Neves Schmitz Gonçalves, Departamento de Engenharia de

Transportes, UFRJ/COPPE. (daniel.schmitz.jf@gmail.com)

${ }^{6}$ Fabiana do Couto Assumpccão1, Departamento de Engenharia de

Transportes, UFRJ/COPPE. (fabianaassumpcao@poli.ufrj.br)
}

Manuscrito recebido em 19/04//2016 e aprovado para publicação em $31 / 08 / 2016$

Este artigo é parte de TRANSPORTES v. 24, n. 3, 2016. ISSN: 2237-1346 (online). DOI:10.4237/transportes.v24i3.1137 de dióxido de carbono $\left(\mathrm{CO}_{2}\right)$, além de custos e segurança (Behendes et al., 2008 e Mckinnon, 2010).

Nesse contexto, o desafio da sociedade consiste em fomentar a entrega, coleta e transferência de cargas, sem comprometer a mobilidade urbana, promovendo o menor impacto ambiental possível. Além disso, o estudo apresentado por Industry Steering (2002) declara a dificuldade de se considerar aspectos socioambientais no planejamento do TUC. Portanto, aprimorar o entendimento desse problema justifica a realização deste trabalho.

Objetiva-se com tal estudo identificar, por meio de uma revisão bibliográfica sistemática, quais os principais desafios enfrentados pelo TUC, bem como os agentes para a sua implantação. Entende-se como agentes o poder público ou a iniciativa privada, ambos com a participação da sociedade. Como objetivos secundários, pretende-se identificar as boas práticas que estão sendo adotadas a fim de solucionar tais desafios, considerando os aspectos socioambientais.

Após esta introdução, o trabalho está dividido em quatro seções. A seção dois apresenta o procedimento adotado e a estrutura da pesquisa. A seção três apresenta a aplicação do procedimento e os resultados encontrados, com ênfase nas fases de planejamento, execução e divulgação. $\mathrm{Na}$ seção quatro apresentam-se as análises. Finalmente, a seção cinco traz as conclusões, as limitações e as recomendações para trabalhos futuros. 


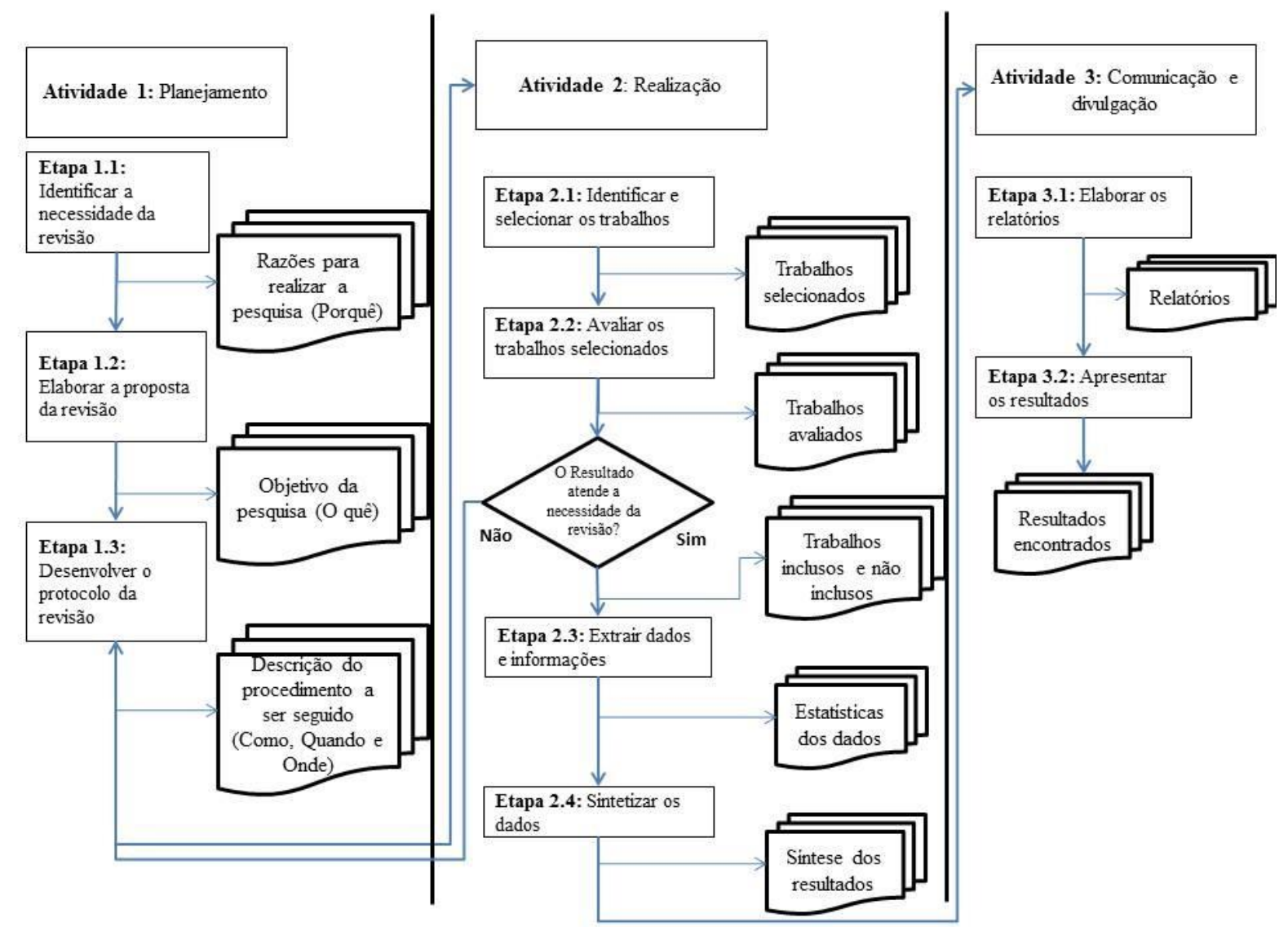

Figura 1. Procedimento de revisão bibliográfica sistemática adotado neste estudo

\section{PROCEDIMENTO E ESTRUTURA DA REVISÃO BIBLIOGRÁFICA SISTEMÁTICA}

A revisão bibliográfica sistemática visa realizar a atualização do estado da arte de uma determinada área de conhecimento específica (Rowley e Slack, 2004). Nesse contexto, o aumento da confiabilidade dos resultados e a possibilidade de redução de erros passam pela utilização de procedimentos sistemáticos (Cook et al., 1997; Bereton et al., 2007). Além disso, Loureiro et al. (2016) destacam positivamente o uso do método sistemático, tendo em vista a sua característica formal e replicável e ressaltam sua capacidade de consolidar grande volume de informações, analisá-los e apresentá-los de forma inteligível.

Para o alcance dos resultados preconizados, Tranfield, Denyer e Smart (2003) apontam uma divisão da revisão bibliográfica sistemática em três etapas: (1) Planejamento da revisão; (2) Realização da revisão e (3) Comunicação e divulgação da revisão. A Figura 1 sintetiza uma proposta de procedimento para a revisão bibliográfica sistemática a ser apresentada neste artigo.

A Atividade de Planejamento consiste em três etapas: (1) identificar a necessidade da revisão; (2) elaborar a proposta da revisão e (3) desenvolver o protocolo da revisão.

A Atividade de Realização consiste em quatro etapas: (1) Identificar e selecionar os trabalhos; (2) Avaliar os trabalhos selecionados; (3) Extrair dados e informações; e (4) Sintetizar os dados.

A Atividade de Comunicação e Divulgação consiste em duas etapas: (1) Elaborar os relatórios e (2) Apresentar os resultados.

\section{APLICAÇÃO DO PROCEDIMENTO E RESULTADOS ENCONTRADOS}

A seguir passa-se a aplicação do procedimento ( $\mathrm{Fi}$ gura 1) e a apresentação dos resultados encontrados.

\subsection{Atividade 1 - Planejamento}

Nesta seção, descreve-se o planejamento da revisão bibliográfica sistemática, primeira atividade do procedimento.

\subsubsection{Etapa 1.1: Identificar a necessidade da revisão}

O TUC é uma atividade que envolve recursos econômicos e financeiros (custos para um dado nível de serviço) e provoca impactos ambientais e sociais, sendo realizadas por entidades públicas e privadas (com a participação da sociedade), em áreas urbanas e mais adensadas. Para que atinja seus objetivos de forma efetiva, o TUC necessita de soluções que respondam às necessidades da demanda, de forma eficiente do ponto de vista econômico e financeiro, causando os menores impactos socioambientais (Ibeas et al., 2012).

Dessa forma, conhecer os principais desafios enfrentados pelos TUC, bem como as boas práticas que podem ser implantadas a fim de solucionar tais desafios, se faz necessário para o seu aprimoramento. 


\subsubsection{Etapa 1.2: Elaborar a proposta de revisão}

A presente revisão bibliográfica sistemática tem por objetivo identificar os principais desafios enfrentados pelo TUC, considerando os agentes para sua implantação (poder público ou iniciativa privada). Como objetivos secundários, pretende-se identificar quais soluções estão sendo aplicadas, a fim de vencer tais desafios, considerando os aspectos socioambientais.

\subsubsection{Etapa 1.3: Desenvolver o protocolo de revisão}

A identificação dos estudos foi realizada na base de dados on line Science Direct tendo em vista que esta base indexa periódicos classificados pelo Qualis da CAPES nas diversas áreas das engenharias e também outros periódicos com relevante fator de impacto e índice h que não constam do Qualis. Além disso, os estudos passam por uma avaliação criteriosa, garantindo a qualidade do material consultado.

A seleção ocorreu mediante o uso de palavras chaves, urban freight transport, urban freight, freight transport (transporte urbano de carga, carga urbana e transporte de carga). A escolha de tais palavras chaves está relacionada ao objetivo do presente estudo que visa identificar os principais desafios e as possíveis boas práticas relacionadas à operação de transporte urbano de carga. Além disso, tal escolha baseou-se na análise das palavras chaves de artigos identificados por meio de um levantamento preliminar a realização deste estudo (7 artigos). Os operadores lógicos "ou" e "e", foram utilizados para facilitar a combinação dos critérios de identificação e seleção dos trabalhos. O caminho utilizado para buscar as palavras chaves foi aplicado no título e no resumo dos artigos.

Considerou-se de 2003 a 2014, como o período para realização da pesquisa, considerando assim os últimos 11 anos de publicação nesta área de conhecimento. Por se entender que o tema é importante para a economia de qualquer país, não foi adotada uma delimitação específica em termos geográficos.

Baseado em Nord et al. (1995) e Ngai e Wat, (2002) e a fim de assegurar a qualidade dos estudos a serem considerados, a busca (identificação dos artigos) foi restrita a artigos publicados em periódicos internacionais indexados e revisados por pares. O critério para seleção dos estudos identificados baseou-se exclusivamente na língua em que o artigo foi escrito (Inglês).

Quanto aos critérios de inclusão e exclusão dos trabalhos, levou-se em consideração o conteúdo do estudo. Todos os trabalhos que descrevessem desafios enfrentados ou boas práticas a serem implementadas ao TUC, que consideraram de alguma forma, aspectos socioambientais, foram incluídos nesta revisão bibliográfica sistemática.

Mediante um banco de dados, foi feito o registro das informações levantadas, a fim de facilitar a classificação, averiguação e avaliação dos estudos empregados nesta pesquisa.

\subsection{Atividade 2 - Realização}

Após o planejamento, é a realizada a revisão bibliográfica sistemática.

\subsubsection{Etapa 2.1: Identificar e selecionar trabalhos}

A identificação e seleção dos estudos seguiram os critérios apresentados no subitem 3.1.3, resultando na identificação de 41 artigos cuja leitura dos resumos foi realizada por, pelo menos, dois autores, tal como recomendado por Thomé et al. (2016) e vom Brocke et al. (2009). O processo de seleção foi iterativo, de modo a buscar a concordância entre os pesquisadores, sendo selecionados 29 artigos para a leitura completa.

\subsubsection{Etapa 2.2: Avaliar os trabalhos selecionados}

A avaliação dos estudos seguiu os critérios descritos no subitem 3.1.3. A leitura completa de cada um dos artigos foi realizada por pelo menos três autores deste estudo. Ao final, realizou-se um painel para discussão do conteúdo dos artigos. Tal painel teve duração de aproximadamente quatro horas e todos os autores puderam apresentar os resultados encontrados. Após a realização desta atividade, optou-se por incluir os 29 artigos selecionados no processo de revisão bibliográfica sistemática. Tais artigos estão distribuídos por seis periódicos científicos diferentes (Figura 2), com maior concentração em: Procedia Social and Behavioral Science (19 artigos).

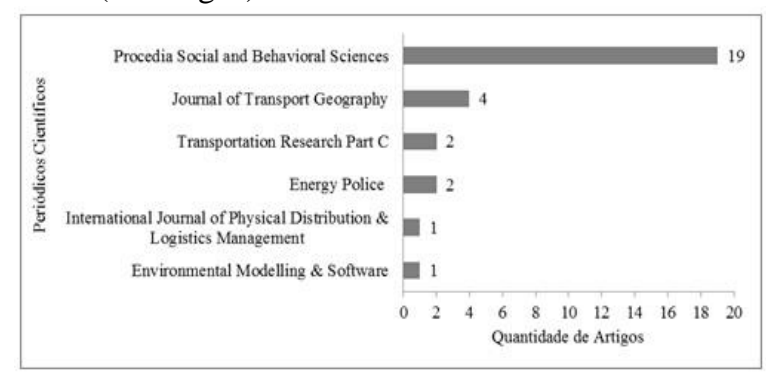

Figura 2. Distribuição dos artigos selecionados por periódicos

Tendo em vista que a revisão bibliográfica sistemática considerou apenas artigos científicos e que isso representa apenas a visão acadêmica do tema abordado neste estudo, optou-se por realizar uma revisão bibliográfica narrativa complementar. Sendo assim, uma alteração no protocolo já descrito no subitem 3.1.3 e uma segunda busca foram realizadas, a fim de garantir a inclusão de referências que pudessem refletir a realidade brasileira.

Para esta revisão, o único critério adotado foi quanto ao tipo de trabalho. Buscou-se identificar relatórios de instituições ligadas a atividade de transporte, internacionais e nacionais. Além disso, buscou-se identificar também revistas nacionais relacionadas à temática transporte, que em seu conteúdo, abordassem boas práticas para o transporte de cargas. Essa decisão foi tomada tendo em vista que se pretende direcionar os resultados desse estudo para o Brasil e que este, não foi identificado por meio da revisão bibliográfica sistemática.

Sendo assim, além dos 29 artigos selecionados no processo de revisão bibliográfica sistemática, foram incluídos também 26 relatórios de instituições internacionais e nacionais (13 relatórios cada). Das instituições nacionais, pode-se destacar a Confederação Nacional do Transporte (CNT), o Instituto de Transporte e Logística (ITL), o Conselho Empresarial Brasileiro para Desenvolvimento Sustentável (CEBDS), a Confederação Nacional das Indústrias (CNI) e o Painel Brasileiro de Mudanças Climáticas (PBMC). 
Além disso, considerou-se também a inclusão de 21 exemplares de revistas brasileiras, sendo 15 exemplares da revista Transporte Moderno, cinco exemplares da revista CNT (Confederação Nacional dos Transportes) - Transporte Atual e um Exemplar da Revista Negócios em Transporte. Totalizando assim, um total de 76 estudos para esta revisão bibliográfica sistemática.

\subsubsection{Etapa 2.3: Extrair dados e informações}

Os dados estatísticos relacionados aos trabalhos inclusos na realização da revisão bibliográfica sistemática encontram-se sintetizados nas Figuras 3, 4 e 5. Para as informações demonstradas nas Figuras 4 e 5 foi possível incluir, também, as estatísticas relacionadas à revisão narrativa complementar.

Em relação aos termos utilizados para identificação dos trabalhos, observa-se maior concentração (23) de artigos localizados por meio dos termos: Urban Freight Transport (12) e Urban Freight (11) (Figura 3).

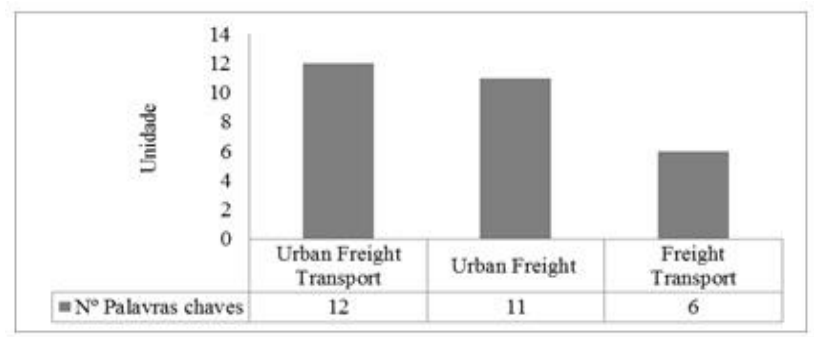

Figura 3. Distribuição dos artigos com base nas palavras-chaves usadas sua seleção

Em relação à abrangência temporal, verificou-se o aumento no número de artigos publicados nos anos de 2010 (6), 2012 (11) e 2014 (7). Com base nos critérios descritos no subitem 3.1.3, não foram encontrados artigos nos anos de 2005, 2007, 2008 e 2009. Em relação aos estudos oriundos da revisão narrativa complementar (relatórios nacionais e internacionais e revistas nacionais), verificou-se que as revistas brasileiras estão distribuídas entre os anos de 2008 a 2015. Quanto aos relatórios, os internacionais estão distribuídos entre os anos de 2002 a 2014 e os nacionais entre os anos de 2007 a 2016 (Figura 4).

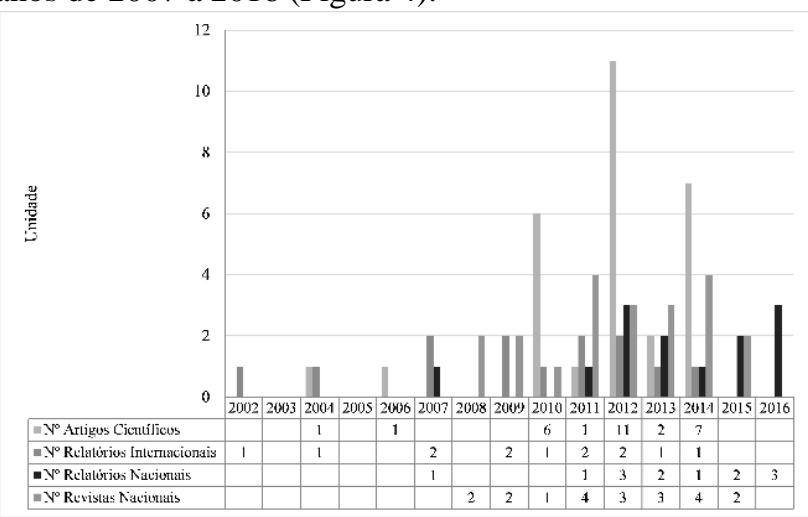

Figura 4. Distribuição dos artigos com base na abrangência temporal

Em relação à abrangência geográfica, verificou-se que os estudos inclusos pertencem a quase todos os continentes do planeta. Nota-se maior concentração dos trabalhos na América do Sul (34) e na Europa (27), seguido da Oceania com oito estudos. Em seguida a América do Norte com cinco estudos. O continente asiático contribuiu com dois estudos (Figura 5).

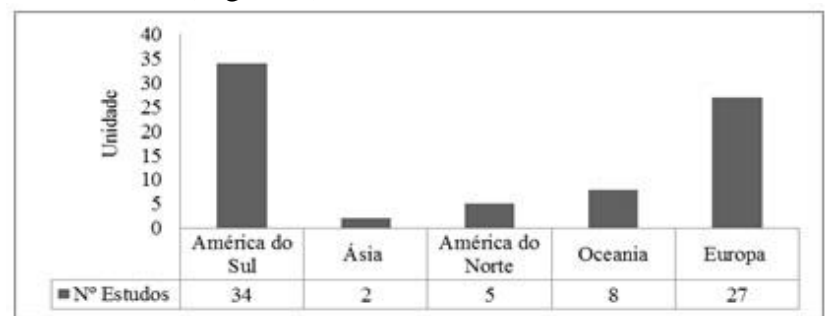

Figura 5. Distribuição dos artigos com base na abrangência geográfica

\subsubsection{Etapa 2.4: Sintetizar os dados}

A etapa 2.4 trata da síntese dos dados. Nesta etapa foi elaborada a Tabela 1A contendo uma relação de todos os desafios identificados nos estudos e a Tabela 1B contendo uma relação de todas as boas práticas identificadas nos estudos.

\subsection{Atividade 3 - Comunicação e Divulgação}

A comunicação e divulgação dos resultados da revisão bibliográfica sistemática foram feitas por meio da elaboração deste artigo, resumindo assim, as Etapas 1 (Elaborar relatório) e 2 (Apresentar resultados) estabelecidas no procedimento (Figura 1).

\section{ANÁLISE DOS RESULTADOS ENCONTRADOS}

Por meio dos resultados da revisão bibliográfica sistemática, foi possível identificar os desafios enfrentados pelo TUC, bem como as boas práticas que podem ser adotadas, a fim de contorná-los, privilegiando aspectos socioambientais. Além disso, identificaram-se também os agentes de sua implantação (poder público ou iniciativa privada).

As boas práticas identificadas estão relacionadas ao planejamento e a gestão da operação do transporte de carga e poderão auxiliar os tomadores de decisão quanto à sua adoção. Tais boas práticas são capazes de promover ganhos socioambientais, demonstrando assim a relevância deste estudo.

Por meio de uma revisão bibliográfica, seguindo um procedimento elaborado exclusivamente para este estudo, foi possível identificar 16 desafios para o TUC, dentre eles, destacam-se: (1) a intensificação dos congestionamentos de tráfego $(79 \%)$; (2) a restrição espaço-temporal da circulação de veículos de carga (76\%); (3) a promoção da redução dos impactos ambientais e sociais decorrentes da atividade de transporte de carga (69\%) e (4) a falta de interação entre os atores envolvidos (transportadoras, clientes, operadores de carga, poder público, etc.) (52\%).

Em relação às boas práticas para o TUC, identificaram-se 26 boas práticas, dentre elas, destacam-se: (1) implantação de centros de distribuição em áreas urbanas (38\%); a utilização de diferentes tipos veículos para realização de entregas e coletas (31\%) e a utilização de sistemas de informação para rastreamento e acompanhamento de frota $(28 \%)$. 
Identificando os Desafios e as Boas Práticas Para o Transporte Urbano de Cargas, Por Meio de Uma Revisão Bibliográfica Sistemática

Tabela 1a. Relação dos desafios identificados na revisão bibliográfica sistemática.

\begin{tabular}{|c|c|c|c|}
\hline Desafios Identificados & Agente & Aspecto & Referências \\
\hline $\begin{array}{l}\text { Antipatia da população em relação à operação do transporte de } \\
\text { cargas }\end{array}$ & $\begin{array}{l}\text { Iniciativa privada e poder pú- } \\
\text { blico (parceria) }\end{array}$ & Social & Quezada e Romero, 2010; Leonardi et al., 2012 ** \\
\hline Demasiada extensão do deslocamento principal & Iniciativa privada & Ambiental e Econômico & $\begin{array}{l}\text { Lindholm e Behrends, } 2012 \text { **; Bhusiri } \text { et al., 2014; Comendador } \text { et al., 2012; Do- } \\
\text { mínguez et al., 2012; Zanni e Bristow, 2010; Figliozzi, 2011** }\end{array}$ \\
\hline Dificuldade de renovação da frota & $\begin{array}{l}\text { Iniciativa privada e poder pú- } \\
\text { blico (parceria) }\end{array}$ & Ambiental e Econômico & Ozen e Tuydes-Yaman, 2013; Lindholm e Behrends, 2012** \\
\hline $\begin{array}{l}\text { Dificuldade em quantificação dos custos e da demanda por } \\
\text { transporte em áreas urbanas }\end{array}$ & Iniciativa privada & Econômico & $\begin{array}{l}\text { Ozen e Tuydes-Yaman, 2013; Balm et al., 2014; Donnelly et al., 2012; Ibeas } \text { et al., } \\
\text { 2012; Timms, 2014; Marquez e Salim, 2007; Rodrigues et al., } 2010\end{array}$ \\
\hline $\begin{array}{l}\text { Dificuldade na obtenção de dados reais sobre a operação de trans- } \\
\text { porte }\end{array}$ & Iniciativa privada & Econômico & $\begin{array}{l}\text { Ballantyne } \text { et al., 2013; Lindholm e Behrends, 2012**; Balm et al., 2014; Donnelly } \\
\text { et al., 2012; Ibeas et al., 2012; Leonardi et al., 2014; Lindholm, 2010; Zanni e } \\
\text { Bristow, 2010; Marquez e Salim, 2007; Figliozzi, 2011** }\end{array}$ \\
\hline $\begin{array}{l}\begin{array}{l}\text { Escassez ou inadequação do uso de locais para realização de carga } \\
\text { e descarga }\end{array} \\
\end{array}$ & Poder público & $\begin{array}{l}\text { Social, Ambiental e } \\
\text { Econômico }\end{array}$ & $\begin{array}{l}\text { Ballantyne } \text { et al., 2013; Bhusiri et al., 2014; Comendador } \text { et al., 2012; Browne } \text { et al., } \\
\text { 2012**; Janjevic e Ndiaye, 2014; Lindholm, 2012; Timms, } 2014\end{array}$ \\
\hline $\begin{array}{l}\text { Falta de equipamentos adequados para a realização da } \\
\text { descarga do veículo }\end{array}$ & Iniciativa privada & Econômico & Filippi et al., 2010** \\
\hline $\begin{array}{l}\text { Falta de interação entre os atores envolvidos (transportadoras, cli- } \\
\text { entes, operadores de carga, poder público, etc) }\end{array}$ & $\begin{array}{l}\text { Iniciativa privada e poder pú- } \\
\text { blico (parceria) }\end{array}$ & $\begin{array}{l}\text { Social, Ambiental e } \\
\text { Econômico }\end{array}$ & $\begin{array}{l}\text { Crainic } \text { et al., } 2014 \text { **; Ballantyne } \text { et al., 2013; Lindholm e Behrends, 2012**; Balm } \\
\text { et al., 2014; Quezada e Romero, 2010; Comendador } \text { et al., 2012; Domínguez et al., } \\
\text { 2012; Leonardi } \text { et al., 2012**; Leonardi } \text { et al., 2014; Lindholm, 2010; Lindholm, } \\
\text { 2012; Quak, 2012; Suksri e Raicu, 2012**; Timms, 2014; Rodrigues } \text { et al., 2010 }\end{array}$ \\
\hline Falta de priorização da mobilidade de carga & Poder público & $\begin{array}{l}\text { Social, Ambiental e } \\
\text { Econômico }\end{array}$ & $\begin{array}{l}\text { Crainic et al., 2014**; Ballantyne et al.., 2013; Lindholm e Behrends, 2012**; } \\
\text { Janjevic e Ndiaye, 2014; Lindholm, } 2010\end{array}$ \\
\hline Falta de segurança da carga ou valores transportados & Iniciativa privada & Econômico & Leonardi et al., 2014 \\
\hline Intensificação do desgaste do pavimento das vias e calçadas & Poder público & Econômico & $\begin{array}{l}\text { Crainic et al., 2014**; Ballantyne } \text { et al., 2013; Quezada e Romero, 2010; Ibeas et al., } \\
\text { 2012; Janjevic e Ndiaye, 2014; Quak, 2012; Suksri e Raicu, 2012** }\end{array}$ \\
\hline Intensificação dos congestionamentos de tráfego & Poder público & $\begin{array}{l}\text { Social, Ambiental e } \\
\text { Econômico }\end{array}$ & $\begin{array}{l}\text { Crainic et al., 2014**; Ballantyne et al., 2013; Lindholm e Behrends, 2012**; As- } \\
\text { chauer e Starkl, 2010; Balm et al., 2014; Quezada e Romero, 2010; Bhusiri et al., } \\
\text { 2014; Browne } \text { et al., 2012**; Comendador } \text { et al., 2012; Domínguez et al., 2012; Fi- } \\
\text { lippi et al., 2010**; Feliu e Salanova, 2012; Ibeas et al., 2012; Janjevic e Ndiaye, } \\
\text { 2014; Leonardi et al., 2012**; Leonardi et al., 2014; Lindholm, 2010; Lindholm, } \\
\text { 2012; Suksri e Raicu, 2012**; Zanni e Bristow, 2010; Marquez e Salim, 2007; Fi- } \\
\text { gliozzi, 2011**; Dinwoodie, 2006 }\end{array}$ \\
\hline Limitação na formação e qualificação de mão-de-obra & Iniciativa privada & Ambiental e Social & Ozen e Tuydes-Yaman, 2013; Lindholm, 2010; Zanni e Bristow, 2010 \\
\hline $\begin{array}{l}\text { Promoção da redução dos impactos ambientais e sociais } \\
\text { decorrentes da atividade do transporte de carga }\end{array}$ & $\begin{array}{l}\text { Iniciativa privada e poder pú- } \\
\text { blico (parceria) }\end{array}$ & Ambiental e Social & $\begin{array}{l}\text { Crainic } \text { et al., 2014**; Ozen e Tuydes-Yaman, 2013; Lindholm e Behrends, 2012**; } \\
\text { Aschauer e Starkl, 2010; Quezada e Romero, 2010; Browne } \text { et al., 2012**; Comen- } \\
\text { dador } \text { et al., 2012; Filippi } \text { et al., 2010**; Feliu e Salanova, 2012; Ibeas et al., 2012; } \\
\text { Leonardi } \text { et al., 2012**; Lindholm, 2010; Lindholm, 2012; Suksri e Raicu, 2012**; } \\
\text { Timms, 2014; Zanni e Bristow, 2010; Walker e Manson, 2014; Marquez e Salim, } \\
\text { 2007; Figliozzi, 2011**; Dinwoodie, 2006; }\end{array}$ \\
\hline Realização de viagens com a ocupação parcial ou nula dos veículos & Iniciativa privada & Ambiental e Econômico & Ozen e Tuydes-Yaman, 2013; Browne et al., 2012**; Rodrigues et al., 2010 \\
\hline Restrição espaço-temporal da circulação de veículos de carga & Poder público & $\begin{array}{l}\text { Social, Ambiental e } \\
\text { Econômico }\end{array}$ & $\begin{array}{l}\text { Crainic et al., 2014**; Ballantyne et al, 2013; Lindholm e Behrends, 2012**; As- } \\
\text { chauer e Starkl, 2010; Balm et al, 2014; Quezada e Romero, 2010; Bhusiri et al, 2014; } \\
\text { Domínguez et al, 2012; Filippi et al, 2010**; Ibeas et al, 2012; Janjevic e Ndiaye, } \\
\text { 2014; Leonardi et al, 2014; Lindholm, 2010; Lindholm, 2012; Quak, 2012; Suksri e } \\
\text { Raicu, 2012**; Timms, 2014; Zanni e Bristow, 2010; Marquez e Salim, 2007; Figli- } \\
\text { ozzi, 2011**; Dinwoodie, 2006; Walker e Manson, 2014 }\end{array}$ \\
\hline
\end{tabular}

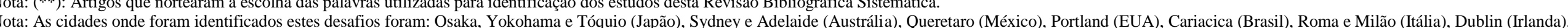

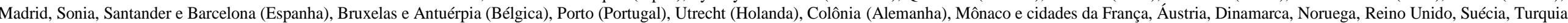
e região do mar Báltico. 
Boas Práticas Identificadas

Ampliação da rede ferroviária em áreas urbanas

Aumento do preço de combustíveis e taxação para emissões de $\mathrm{CO} 2$

Fiscalização física e eletrônica de estacionamentos de carga e descarga

Implantação de centros de consolidação de carga em áreas urbanas

Implantação de centros de distribuição em áreas urbanas

Implantação de equipamento de controle das emissões nos veículos

Implantação de sistema de gestão integrado dos modos de transporte

Manutenção preventiva dos veículos

Otimização da ocupação do veículo

Otimização da operação de carga e descarga com utilização de equipamentos motorizados

Otimização das rotas

Promoção de melhoria da aerodinâmica dos veículos

Promoção de uma melhor gestão de tráfego

Realização de coleta e distribuição noturna Realização de coleta e distribuição noturna
mais limpos - transferência modal
maisferência do transporte carga para modos Redução do peso dos veículos

Renovação e modernização da frota

Restrição do tráfego de veículos pesados em área urbana

Revitalização e manutenção preventiva regular das rodovias (via
urbana)

Treinamento de motoristas (Eco-driving)

Utilização de faixas exclusivas para veículos de carga

Tabela 1b. Relação das boas práticas identificadas na revisão bibliográfica sistemática

\begin{tabular}{|c|c|c|}
\hline Agente & Aspecto & Referências \\
\hline Poder público & $\begin{array}{c}\text { Econômico, Social e } \\
\text { Ambiental }\end{array}$ & Dinwoodie, 2006; Dablanc, 2009 \\
\hline Poder público & $\begin{array}{c}\text { Econômico, Social e } \\
\text { Ambiental }\end{array}$ & Browne et al., 2012** \\
\hline Poder público & $\begin{array}{l}\text { Econômico, Social e } \\
\text { Ambiental }\end{array}$ & Browne et al., 2012**; Filippi et al., 2010**; BESTUFS, 2007 ; Dablanc, 2009 \\
\hline Iniciativa privada & $\begin{array}{c}\text { Econômico, Social e } \\
\text { Ambiental }\end{array}$ & $\begin{array}{l}\text { Balm et al., 2014; Timms, 2014; Crainic et al., 2014**; Zanni e Bristow, 2010; Da- } \\
\text { blanc, } 2011\end{array}$ \\
\hline Iniciativa privada & $\begin{array}{l}\text { Econômico, Social e } \\
\text { Ambiental }\end{array}$ & $\begin{array}{l}\text { Browne et al., 2012**; Domínguez et al., 2012; Filippi et al., 2010**; } 15 \text { Feliu e Sa- } \\
\text { lanova, 2012; Leonardi et al., 2012**; Leonardi et al., 2014; Lindholm, 2010; Lin- } \\
\text { dholm, 2012; Quak, 2012; Suksri e Raicu, 2012**; Zanni e Bristow, 2010; Bestufs, } \\
\text { 2007; Bestfact, 2004; Dablanc, } 2011\end{array}$ \\
\hline Iniciativa privada & Ambiental e Social & Figliozzi, 2011** \\
\hline $\begin{array}{l}\text { Iniciativa privada e poder pú- } \\
\text { blico (parceria) }\end{array}$ & Econômico, Social e Ambiental & $\begin{array}{l}\text { Lindholm e Behrends, 2012**; Aschauer e Starkl, 2010; Ballantyne et al., 2013; Que- } \\
\text { zada e Romero, 2010; Browne et al., 2012**; Dablanc, } 2011\end{array}$ \\
\hline Iniciativa privada & Econômico, Social e Ambiental & $\begin{array}{l}\text { Filippi } \text { et al., 2010**; Transporte Moderno } \mathrm{n}^{\circ} \text { 456, 2013; CAI-Asia, 2011; The World } \\
\text { Bank, 2012; CNT, 2007; CNT, } 2012\end{array}$ \\
\hline Iniciativa privada & Econômico, Social e Ambiental & $\begin{array}{l}\text { Zanni e Bristow, 2010; CAI-Asia, 2011; Kamakaté e Schipper, 2009; CNT, } 2016 \text { (b); } \\
\text { CNT, 2015; CNT Transporte Atual no 239, 2015; CNI, } 2011\end{array}$ \\
\hline Iniciativa privada & Econômico, Social e Ambiental & Filippi et al., 2010**; Bestufs, 2007 \\
\hline Iniciativa privada & Econômico, Social e Ambiental & $\begin{array}{l}\text { Crainic et al., 2014; Balm et al., 2014; Bhusiri et al., 2014; Leonardi et al., 2014; } \\
\text { Timms, 2014; Industry Steering Committee (2002); Bestfact, 2004; Standing Council } \\
\text { on Transport and Infrastructure, 2012; CAI-Asia, 2011; CNI, 2011; Dablanc, } 2011\end{array}$ \\
\hline Iniciativa privada & Ambiental e Social & $\begin{array}{l}\text { Browne } \text { et al., 2012**; Zanni e Bristow, 2010; The World Bank, 2010; CNT, } 2016 \\
\text { (b); CNT Transporte Atual n }{ }^{\circ} 239,2015\end{array}$ \\
\hline Poder público & Econômico, Social e Ambiental & Ballantyne et al., 2013; Balm et al, 20.14; Timms, 2014 \\
\hline Iniciativa privada & Econômico, Social e Ambiental & Lindholm, 2012; Quak, 2012; Suksri e Raicu, 2012**; Bestufs, 2007; Dablanc, 2011 \\
\hline $\begin{array}{l}\text { Iniciativa privada e poder pú- } \\
\text { blico (parceria) }\end{array}$ & Econômico, Social e Ambiental & $\begin{array}{l}\text { Lindholm e Behrends, 2012**; Aschauer e Starkl, 2010; Browne et al., 2012**; Leo- } \\
\text { nardi et al., 2014; Lindholm, 2012;Zanni e Bristow, 2010; Dinwoodie, } 2006\end{array}$ \\
\hline Iniciativa privada & Ambiental e Social & Suksri e Raicu, 2012**; CNT Transporte Atual no 239, 2015 \\
\hline Iniciativa privada & Econômico, Social e Ambiental & $\begin{array}{l}\text { Filippi et al., 2010**; CAI-Asia, 2011; CNT, } 2016 \text { (a); CNT, } 2016 \text { (b); CNT, } 2016 \\
\text { (c); CNT, 2015; CNT Transporte Atual nº 231, 2014; CNT Transporte Atual no 239, } \\
\text { 2015; NTC \& Logística, } 2012\end{array}$ \\
\hline Poder público & Ambiental e Social & $\begin{array}{l}\text { Aschauer e Starkl, 2010; Domínguez et al., 2012; Filippi et al., 2010**; Lindholm, } \\
\text { 2012; Timms, 2014; Marquez e Salim, 2007; The World Bank, 2010; Dablanc, 2009; } \\
\text { Dablanc, } 2011\end{array}$ \\
\hline Poder público & Econômico, Social e Ambiental & Timms, 2014; Dablanc, 2009 \\
\hline Iniciativa privada & Econômico, Social e Ambiental & $\begin{array}{l}\text { Crainic } \text { et al., 2014**; Balm } \text { et al., 2014; Browne } \text { et al., 2012**; Leonardi } \text { et al., } \\
\text { 2012**; Leonardi } \text { et al., 2014; Lindholm, 2012; Quak, 2012; Timms, 2014; Zanni e } \\
\text { Bristow, 2010; Dablanc, 2011 }\end{array}$ \\
\hline Poder público & Econômico, Social e Ambiental & $\begin{array}{l}\text { Balm et al., 2014; Filippi et al., 2010**; Zanni e Bristow, 2010; Bestufs, 2007; } \\
\text { Industry Steering Committee, 2002; }\end{array}$ \\
\hline
\end{tabular}


Identificando os Desafios e as Boas Práticas Para o Transporte Urbano de Cargas, Por Meio de Uma Revisão Bibliográfica Sistemática Tabela 1b (continuação). Relação das boas práticas identificadas na revisão bibliográfica sistemática Agente

Agente

Utilização de fontes de energia mais limpas

Iniciativa privada

iciativa privada nhamento de frota

Utilização de sistemas de informação para rastreamento e acompa-
Utilização de sistemas de propulsão alternativo

Utilização de veículos com maior eficiência energética

\begin{tabular}{c|c} 
Aspecto & Ambiental e Social \\
& Econômico, Social e Ambiental
\end{tabular}

Utilização de veículos com maior eficiência energética

Nota 1: (**): Artigos que nortearam a escolha das palavras utilizadas para identificação dos estudos desta Revisão Bibliográfica Sistemática.

Nota 2: As cidades para as quais foram sugeridas essas boas práticas são: Osaka, Yokohama e Tóquio (Japão), Sydney e Adelaide (Austrália), Queretaro (México), Portland (EUA), Cariacica (Brasil), Roma e Milão (Itália),

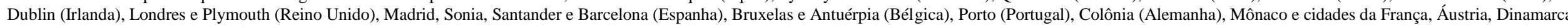

Noruega, Suécia, Holanda, Turquia e região do mar Báltico
(B) et al., 2012*; Quak, 2012; Zanni e Bristow, 2010; Transporte Moderno n 428, 2008; Transporte Moderno $\mathrm{n}^{\circ}$ 429, 2008; Transporte Moderno $\mathrm{n}^{\circ}$ 439, 2010 Transporte Moderno $n^{\circ} 443,2011$; Transporte Moderno n ${ }^{\circ} 454,2012 ; \mathrm{NT}^{\circ}{ }^{\circ} 118$ 2014; Bestufs, 2007; Industry Steering Committee, 2002; Clean Cities, 2014; Clean Cities, 2013; CAI-Asia, 2011; CNT, 2016 (a); CNT Transporte Atual no 227, 2014; CNT Transporte Atual $\mathrm{n}^{\circ} 231,2014$; CNT Transporte Atual $\mathrm{n}^{\circ}$ 232, 2015; CEBDS, 2014; PBMC, 2013; Observatório do Clima, 2015; CNI, 2011; CNI, 2012 1. 2014**; Browne et al., 2012**; Comendador et al., 2012; Leonardi $e$ 014; Quak, 2012; Suksri e Raicu, 2012**; Zanni e Bristow, 2010; Walker e Manson, 2014; Bestufs, 2007; Industry Steering Committee, 2002; Standing Council on Transpot and Infrastructure, 2012; CNT, 2015; CEBDS, 2014

Browne et al., 2012**; Transporte Moderno $\mathrm{n}^{\circ} 443,2011$; Transporte Moderno $\mathrm{n}^{\circ}$ 437, 2009; Transporte Moderno $n^{\circ}$ 428, 2008; Transporte Moderno $\mathrm{n}^{\circ}$ 439, 2010; Transporte Moderno n ${ }^{\circ} 454,2012$; Bestufs, 2007; Bestfact, 2004, Bestufs, 2007; Clea Cities, 2014, Clean Cities, 2013; CAI-Asia, 2011; CNT Transporte Atual nº 223, 2014 CNT Transporte Atual n ${ }^{\circ}$ 232, 2015; CNI, 2012; Dablanc, 2009; Dablanc, 2011 Zanni e Bristow, 2010; NT n ${ }^{\circ} 118$, 2014; Transporte Moderno $\mathrm{n}^{\circ} 458$, 2013; Transpote Moderno $n^{\circ}$ 439, 2010; Transporte Moderno $n^{\circ}$ 437, 2009; Transporte Moderno $\mathrm{n}^{\circ}$ 454, 2012; Transporte Moderno $\mathrm{n}^{\circ}$ 447, 2011; Transporte Moderno $\mathrm{n}^{\circ}$ 444, 2011 Transporte Moderno $\mathrm{n}^{\circ}$ 445, 2011; Transporte Moderno $\mathrm{n}^{\circ}$ 461, 2013; Transporte Mo-

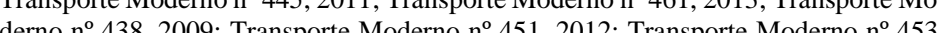
2012; CNT, 2016 (a); NTC \& Logística, 2013; NTC \& Logística, 2012 
Verificou-se que por meio da adoção das boas práticas que privilegiam os aspectos ambientais e sociais é possível obter ganhos econômicos simultaneamente, ainda que seja necessária a realização de um investimento inicial. Das boas práticas identificadas, $81 \%$ podem contribuir de forma positiva, em relação aos aspectos econômicos, ambientais e sociais.

Considerando a influência da boa prática em relação à solução dos desafios, identificou-se que a boa prática que consiste na utilização de sistemas de informação para rastreamento e acompanhamento de frota é capaz de apoiar as empresas na solução de $63 \%$ dos desafios identificados.

Quanto aos agentes de implementação das boas práticas, verificou-se que o poder público pode atuar individualmente por meio de $27 \%$ das boas práticas identificadas, estando relacionado com $31 \%$ dos desafios identificados no TUC. A iniciativa privada poderia contribuir individualmente com a solução de $44 \%$ dos desafios identificados e poderiam atuar por meio de $65 \%$ das boas práticas. Verificou-se que ambos os agentes (iniciativa privada e poder público) podem contribuir conjuntamente de forma positiva com a solução de $25 \%$ dos desafios identificados para o TUC e atuar por meio de $8 \%$ das boas práticas.

Em relação às cidades onde as pesquisas bibliográficas pautaram seus estudos, estes foram realizados em diversas partes do mundo e estão distribuídos nos continentes da Europa, Oceania, Ásia e América do Sul. Dentre as cidades, estudadas, destacam-se as cidades de Tóquio (Japão), Londres (Reino Unido), Madri (Espanha), Barcelona (Espanha), Sidney (Austrália) e Roma (Itália).

\section{CONCLUSÕES, LIMITAÇÕES E RECOMENDAÇÕES PARA TRABALHOS FUTUROS}

O presente trabalho buscou identificar, por meio de uma revisão bibliográfica sistemática, os desafios enfrentados pelo TUC e as boas práticas que podem ser aplicadas a fim de contorná-los, considerando aspectos socioambientais. Além disso, identificaram-se os agentes de implementação das boas práticas.

Como contribuição deste estudo pode-se apontar: (1) a elaboração de um procedimento de revisão bibliográfica sistemática, estabelecido exclusivamente para este estudo e; (2) a identificação de 16 desafios do TUC e 26 boas práticas capazes de solucioná-los, considerando seus impactos socioambientais.

Dentre os desafios identificados, $31 \%$ podem ser solucionados individualmente por parte do poder público, que pode atuar por meio de $27 \%$ das boas práticas. A iniciativa privada pode atuar individualmente na solução de $44 \%$ dos desafios, por meio de $65 \%$ boas práticas. Ambos os agentes podem atuar conjuntamente na solução de $25 \%$ dos desafios, por meio de $8 \%$ das boas práticas. Verificou-se que $81 \%$ das boas práticas podem contribuir de forma positiva em relação aos aspectos econômicos, ambientais e sociais simultaneamente.

A realização dessa pesquisa limitou-se a uma pesquisa bibliográfica. Recomenda-se que seja feita uma pesquisa de campo, a fim de verificar se tais desafios e boas práticas são também considerados na prática da atividade do TUC das cidades.

\section{AGRADECIMENTOS}

Ao Conselho Nacional de Desenvolvimento Científico e Tecnológico $(\mathrm{CNPq})$ pelo apoio financeiro para realização dessa pesquisa.

\section{REFERÊNCIAS}

Allen, J., Browne, M. (2008). Review of Survey Techniques Used in Urban Freight Studies. Report produced as part of the Green Logistics Project: Work Module 9 (Urban Freight Transport), London, U.K., http://www.greenlogistics.org.

Aschauer, G. J. e Starkl, F. (2010) Time4trucks - cooperative time regulation of road freight transportation in urban areas for reducing bottlenecks. Procedia Social and Behavioral Sciences, v. 2, p. 6242-6250. DOI:10.1016/j.sbspro.2010.04.034

Ballantyne, E. E. F., Lindholm, M. e Whiteing, A. (2013). A comparative study of urban freight transport planning: addressing stakeholder needs. Journal of Transport Geography, v.32, p. 93101. DOI: $10.1016 /$ j.jtrangeo.2013.08.013

Balm, S., Browne, M., Leonardi, J. e Quak, H (2014). Developing an Evaluation Framework for Innovative Urban and Interurban Freight Transport Solutions. Procedia - Social and Behavioral Sciences, v.125, p. 386 - 397.

DOI: $10.1016 /$ j.sbspro.2014.01.1482

Behrends, S., Lindholm, M. and Woxenius, J. (2008). The impact of urban freight transport: a definition of sustainability from an actor's perspective. Transportation Planning and Technology, 31 (6), Routledge, Taylor \& Francis Group.

Bereton, P.; Kitchenham, B .A.; Budgen, D.; Turner, M.; Khalil, M. (2007). Lessons from Applying the Sistematic Literature Review Process within the Software Engineering Domain. The Journal of System and Software, v. 80, p.571-583. DOI:10.1016/j.jss.2006.07.009.

Bestfact, Towards a competitive and sustainable European Freight Transport, European Commission, 2004.

Bestufs, Good Practice Guide on Urban Freight Transport, European Commission, 2007.

Bhusiri, N., Qureshi, A. G. e Taniguchi, E. (2014) Application of the Just-In-Time Concept in Urban Freight Transport. Procedia Social and Behavioral Sciences, v. 125, p. $171-185$. DOI: 10.1016/j.sbspro.2014.01.1465

Browne, M., Allen J., Nemoto, T., Patier, D. e Visser, J. (2012) Reducing social and environmental impacts of urban freight transport: A review of some major cities. Procedia - Social and Behavioral Sciences, v. 39, p. $19-33$.

DOI: $10.1016 /$ j.sbspro.2012.03.088

CAI-Asia, Design of Green Freight China Program: Program Design Report, Clean Air Initiative for Asian Cities (CAI-Asia) Center, 2011

CEBDS. Manual de Compras Sustentáveis. Rio de Janeiro, 2014.

Clean Cities, Building Partnerships to Cut, 2014. 
Clean Cities, Guide to Alternative Fuel and Advanced Medium and Heavy-Duty Vehicles, U.S. Department of Energy, 2013.

CNT, Transporte Atual no 223, 2014.

CNT, Transporte Atual nº 227, 2014.

CNT, Transporte Atual no 231, 2014.

CNT, Transporte Atual no 232, 2015.

CNT, Transporte Atual no 239, 2015.

Comendador, J., López-Lambas, M. E. e Monzón, A. (2012) A GPS analysis for urban freight distribution. Procedia - Social and Behavioral Sciences, v. 39, p. $521-533$.

DOI:10.1016/j.sbspro.2012.03.127

Confederação Nacional da Indústria. Estratégias Corporativas de Baixo Carbono: Gestão de Riscos e Oportunidades. Brasília, 2011.

Confederação Nacional da Indústria. Indústria automobilística e sustentabilidade. Associação Nacional dos Fabricantes de Veículos Automotores. Brasília, 2012.

Confederação Nacional do Transporte - CNT, Caminhoneiro amigo do meio ambiente. Brasília, 2012.

Confederação Nacional do Transporte - CNT, Despoluir Programa Ambiental do Transporte. In: Promoção SEST/SENAT - Conteúdo Técnico Escola do Transporte, Brasília, 2007.

Confederação Nacional do Transporte - CNT, Oficina nacional transporte e mudanças climáticas. Disponível em: http://cms.cnt.org.br/Imagens\%20CNT/Site\%202015/Pesquisas \%20PDF/caderno_oficina_nacional_09_09.pdf. Acesso em: 06/07/2016 (a).

Confederação Nacional do Transporte - CNT, Relatório Síntese de Informações Ambientais. Disponível em: http://www.cntdespoluir.org.br/Documents/PDFs/Relatorio_Sint ese_de_Informacoes_Ambientais.pdf. Acesso em: 06/07/2016 (b).

Confederação Nacional do Transporte - CNT, Seminário internacional sobre reciclagem de veículos e renovação de frota. Disponível em:

https://issuu.com/despoluir/docs/seminario_de_reciclagem_veicu los_renovacao_frota/1. Acesso em: 06/07/2016 (c).

Confederação Nacional do Transporte - CNT, Sondagem CNT de eficiência energética no transporte rodoviário de cargas. Brasília, 2015.

Cook, D.J.; Mulrow, C.D.; Haynes, R.B. (1997) Systematic reviews: synthesis of best evidence for clinical decisions. Annals of Internal Medicine, v.126, n.5, pp.376-380. PMID: 9054282

Crainic, T. G., Ricciardi, N. e Storchi, G. (2014) Advanced freight transportation systems for congested urban areas. Transportation Research Part C, v.12. p. 119-137. DOI: $10.1016 /$ j.trc.2004.07.002

Dablanc, L. Freight transport for development toolkit - urban transport. In: The International Bank for Reconstruction and Development. The World Bank 1818 H Street NW, 2009.

Dablanc, L., Patier, D., Feliu, J. G., Augereau, V., Leonardi, J., et al. City Logistics Best Practices: a Handbook for Authorities. In: SUGAR. Sustainable Urban Goods Logistics Achieved by
Regional and Local Policies, Regione Emilia Romagna, Bologna, Itália, pp.276, 2011.

Dinwoodie, J. (2006) Rail freight and sustainable urban distribution: Potential and practice. Journal of Transport Geography, v. 14, p. 309-320.

DOI: $10.1016 /$ j.jtrangeo.2005.06.001

Domínguez, A., Holguín-Veras, J., Ibeas, A. e dell’Olio, L. (2012) Receivers' response to new urban freight policies. Procedia - Social and Behavioral Sciences, v. 54, p. 886 - 896. DOI:10.1016/j.sbspro.2012.09.804

Donnelly, R., Thompson, R. G. e Wigan, M. (2012) Process validation of urban freight and logistics models. Procedia - Social and Behavioral Sciences, v. 39, p. $400-408$. DOI:10.1016/j.sbspro.2012.03.117

Feliu, J. G. e Salanova, J. M. (2012) Defining and evaluating collaborative urban freight transportation systems. Procedia Social and Behavioral Sciences, v. 39, p. $172-183$. DOI:10.1016/j.sbspro.2012.03.099

Figliozzi, M. A. (2011) The impacts of congestion on timedefinitive urban freight distribution networks $\mathrm{CO} 2$ emission levels: Results from a case study in Portland, Oregon. Transportation Research Part C, v. 19, p. 766-778. DOI: $10.1016 /$ j.trc.2010.11.002

Filippi, F., Nuzzolo, A., Comi, A. e Site, P. D. (2010) Ex-ante assessment of urban freight transport policies. Procedia Social and Behavioral Sciences, v. 2, p. 6332-6342. DOI:10.1016/j.sbspro.2010.04.042

Ibeas, A., Moura, J. L., Nuzzolo, A. e Comi, A. (2012) Urban freight transport demand: transferability of survey results analysis and models. Procedia - Social and Behavioral Sciences, v. 54, p. 1068 - 1079. DOI: 10.1016/j.sbspro.2012.09.822

Industry Steering. (2002) Freight Logistics in Australia an Agenda for Action. Industry Steering Committee of the Freight Transport Logistics Industry Action Agenda. Australia.

Janjevic, M. e Ndiaye, A. B. (2014) Development and Application of a Transferability Framework for Microconsolidation Schemes in Urban Freight Transport. Procedia Social and Behavioral Sciences, v. 125, p. $284-296$. DOI:10.1016/j.sbspro.2014.01.1474

Kamakaté, F. e Schipper, L. "Trends in truck freight energy use and carbon emissions in selected OECD countries from 1973 to 2005”, Energy Policy v. 37, pp. 3743-3751, 2009. DOI:10.1016/j.enpol.2009.07.029.

Leonardi, J., Browne, M. e Allen, J. (2012) Before-after assessment of a logistics trial with clean urban freight vehicles: A case study in London. Procedia - Social and Behavioral Sciences, v. 39, p. 146 - 157. DOI:10.1016/j.sbspro.2012.03.097

Leonardi, J., Browne, M., Allen, J., Bohne, S. e Ruesch, M. (2014) Best Practice Factory for Freight Transport in Europe: Demonstrating How 'Good' Urban Freight Cases are Improving Business Profit and Public Sectors Benefits. Procedia - Social and Behavioral Sciences, v. 125, p. $84-98$. DOI:10.1016/j.sbspro.2014.01.1458

Lindholm, M. (2010) A sustainable perspective on urban freight transport: Factors affecting local authorities in the planning procedures. Procedia Social and Behavioral Sciences, v. 2, p. 6205-6216. DOI:10.1016/j.sbspro.2010.04.031 
Lindholm, M. (2012) How local authority decision makers address freight transport in the urban area. Procedia - Social and Behavioral Sciences, v. 39, p. $134-145$.

DOI:10.1016/j.sbspro.2012.03.096

Lindholm, M. e Behrends, S. (2012) Challenges in urban freight transport planning - a review in the Baltic Sea Region. Journal of Transport Geography, v. 22, p. 129-136.

http://dx.doi.org/10.1016/j.jtrangeo.2012.01.001

Loureiro, S. A., Santos Júnior, J. B. S., Noletto A. P. R., Santos, L. S. e Lima Júnior, O. F. "O uso do método de revisão sistemática da literatura na pesquisa em logística, transportes e cadeia de suprimentos. "Transportes v. 24, n. 1, p. 95-106, 2016. DOI: $10.14295 /$ transportes.v24i1.919

Marquez, L. e Salim, V. (2007) Assessing impacts of urban freight measures on air toxic emissions in Inner Sydney. Environmental Modelling \& Software, v. 22, p. 515-525. DOI: $10.1016 /$ j.envsoft.2006.02.007

McKinnon, A., Cullinane, S., Browne, M., Whiteing, A. (2010) Green logistics: improving the environmental sustainability of logistics. Londres, Philadelfia, Nova Delhi. Kogan Page, V. 1.

Negócios em Transporte - ano 12, nº 118, 2014.

Ngai, E. W. T.; Wat, F. K. T. (2002) A literature review and classification of electronic commerce research. Information \& Management. Volume 39, p. 415- 429.

DOI: $10.1016 / \mathrm{S} 0378-7206(01) 00107-0$

Nord, J. H.; Nord, G. D. (1995) MIS Research: Journal status assessment and analysis. Information \& Management. Volume 29, p. 29-42.

NTC\&Logística. Anuário NTC\&Logística Triênio 2011-2012. 2012

NTC\&Logística. Anuário NTC\&Logística Triênio 2012-2013. 2013

Observatório do Clima, Documento síntese: Análise das emissões de GEE no Brasil (1970-2013) e suas implicações para políticas públicas. In: Governos Locais pela Sustentabilidade (ICLEI). [et al.]. - São Paulo, 2015.

Ozen, M. e Tuydes-Yaman, H. (2013) Evaluation of emission cost of inefficiency in road freight transportation in Turkey. Energy Policy, v. 62. p. 625-636.

DOI: $10.1016 / j . e n p o l .2013 .07 .075$

PBMC, Contribuição do Grupo de Trabalho 3 ao Primeiro Relatório de Avaliação Nacional do Painel Brasileiro de Mudanças Climáticas. Sumário Executivo do GT3. Rio de Janeiro, Brasil, 2013.

Quak, H. J. (2012) Improving urban freight transport sustainability by carriers - Best practices from The Netherlands and the EU project CityLog. Procedia - Social and Behavioral Sciences, v. 39, p. 158 - 171. DOI:10.1016/j.sbspro.2012.03.098

Quezada, E. B. e Romero, A. (2010) An urban freight transport index. Procedia Social and Behavioral Sciences, v.2, p. 63126322. DOI:10.1016/j.sbspro.2010.04.040

Rodrigues, V. S, Potter, A. e Naim, M. M. (2010) The impact of logistics uncertainty on sustainable transport operations. International Journal of Physical Distribution \& Logistics Management, v. 40, p. 61-83.

\section{DOI: $10.1108 / 09600031011018046$}

Rowley, J.; Slack, F. (2004) Conducting a literature review. Management Research News. Volume 27, nº 6, p. 31-39,

Standing Council on Transpot and Infrastructure, The National Land Freight Strategy, 2012.

Suksri, J. e Raicu, R. (2012) Developing a conceptual framework for the evaluation of urban freight distribution initiatives. Procedia - Social and Behavioral Sciences, v. 39, p. 321 - 332. DOI:10.1016/j.sbspro.2014.01.1482

The World Bank, Guangzhou Green Trucks: "Truck GHG Emission Reduction Pilot Project", Australian Government (AusAID), 2012.

The World Bank, Guangzhou Green Trucks: "Truck GHG Emission Reduction Pilot Project", Australian Government (AusAID), 2010.

Thomé, A.M., Scavarda, L. F., Scavarda, A.J. (2016): Conducting systematic literature review in operations management, Production Planning \& Control, DOI: 10.1080/09537287.2015.1129464.

Timms, P. (2014) Transferability of urban freight transport measures: A case study of Cariacica (Brazil). Research in Transportation Business \& Management. Article in press. http://dx.doi.org/10.1016/j.rtbm.2014.02.001

Tranfield, D., Denyer, D. \& Smart, P. (2003). Towards a methodology for developing evidence-informed management knowledge by means of systematic review. British Journal of Management, 14, 207-222.

Transporte Moderno - ano 45, $\mathrm{n}^{\circ}$ 428, 2008.

Transporte Moderno - ano 45, nº 429, 2008.

Transporte Moderno - ano 46, $\mathrm{n}^{\circ}$ 437, 2009.

Transporte Moderno - ano 46, n 438, 2009.

Transporte Moderno - ano 47, $n^{\circ}$ 439, 2010.

Transporte Moderno - ano 48, $\mathrm{n}^{\circ}$ 443, 2011.

Transporte Moderno - ano 48, $\mathrm{n}^{\circ}$ 444, 2011.

Transporte Moderno - ano 48, n 445, 2011.

Transporte Moderno - ano 48, $\mathrm{n}^{\circ}$ 447, 2011.

Transporte Moderno - ano 49, $\mathrm{n}^{\circ}$ 451, 2012.

Transporte Moderno - ano 49, $\mathrm{n}^{\circ}$ 453, 2012.

Transporte Moderno - ano 49, $\mathrm{n}^{\circ}$ 454, 2012.

Transporte Moderno - ano 49, $\mathrm{n}^{\circ}$ 456, 2013.

Transporte Moderno - ano 50, n 458, 2013.

Transporte Moderno - ano 50, $\mathrm{n}^{\circ}$ 461, 2013.

Vom Brocke, J., A. Simons, B. Niehaves, K. Riemer, R. Plattfaut, and A. Cleven. 2009. "Reconstructing the Giant: On the Importance of Rigour in Documenting the Literature Search 
Process." 17th European Conference on Information Systems,

Verona, June 8-10.

Walker, G. e Manson, A. (2014) Telematics, urban freight logistics and low carbon road networks. Journal of Transport Geography, v. 37, p. 74-81.

DOI: $10.1016 /$ j.jtrangeo.2014.04.006

Zanni, A. M. e Bristow, A. L. (2010) Emissions of CO2 from road freight transport in London: Trends and policies for long run reductions. Energy Policy, v. 38, p. 1774-1786.

DOI: $10.1016 /$ j.enpol.2009.11.053 